\title{
Serum metabolomics using ultra performance liquid chromatography coupled to mass spectrometry in lactating dairy cows following a single dose of sporidesmin
}

\author{
Zoe M. Matthews ${ }^{1} \cdot$ Patrick J. B. Edwards ${ }^{1} \cdot$ Ariane Kahnt $^{2} \cdot$ Mark G. Collett $^{1}$ - Jonathan C. Marshall ${ }^{1}$. \\ Ashton C. Partridge ${ }^{2} \cdot$ Scott J. Harrison ${ }^{2} \cdot$ Karl Fraser $^{3} \cdot$ Mingshu Cao $^{3} \cdot$ Peter J. Derrick ${ }^{2}$
}

Received: 24 October 2017 / Accepted: 29 March 2018 / Published online: 17 April 2018

(c) The Author(s) 2018

\begin{abstract}
Introduction Photosensitization is a common clinical sign in cows suffering from liver damage caused by the mycotoxin sporidesmin. This disease, called facial eczema (FE), is of major importance in New Zealand. Current techniques for diagnosing animals with subclinical sporidesmin-induced liver damage (i.e. without photosensitization) are nonspecific. In addition, little is known of the mechanisms involved in sporidesmin resistance, nor the early effects seen following low-dose sporidesmin intoxication.

Objective The objective of this study was to identify individual metabolites or metabolic profiles that could be used as serum markers for early stage FE in lactating cows.

Methods Results are presented from a 59-day sporidesmin challenge in Friesian-cross dairy cows. Serum metabolite profiles were obtained using reversed phase ultra-performance liquid chromatography (UPLC) electrospray ionization mass spectrometry (MS) and UPLC tandem MS. Multivariate and time series analyses were used to assess the data.

Results Statistical analysis, both with and without the temporal component, could distinguish the profiles of animals with clinical signs from the others, but not those affected subclinically. An increase in the concentrations of a combination of taurine- and glycine-conjugated secondary bile acids (BAs) was the most likely cause of the separation. This is the first time that MS methods have been applied to FE and that bile acids changes have been detected in cattle exposed to sporidesmin. Conclusions It is well known that BA concentrations increase during cholestasis due to damage to bile ducts and leakage of the bile. This is the first study to investigate metabolomic changes in serum following a sporidesmin challenge. Further work to establish the significance of the elevation of individual BAs concentrations in the serum of early-stage sporidesminpoisoned cows is necessary.
\end{abstract}

Keywords Sporidesmin $\cdot$ Facial eczema $\cdot$ Mass spectrometry $\cdot$ Metabolomics $\cdot$ Bile acid $\cdot$ Dairy cow $\cdot$ Serum

\section{Introduction}

Peter J. Derrick-Deceased.

Electronic supplementary material The online version of this article (https://doi.org/10.1007/s11306-018-1358-4) contains supplementary material, which is available to authorized users.

Zoe M. Matthews

z.matthews@massey.ac.nz

$\triangle$ Patrick J. B. Edwards p.j.edwards@massey.ac.nz

1 Massey University, Palmerston North, New Zealand

2 University of Auckland, Auckland, New Zealand

3 AgResearch Grasslands, Palmerston North, New Zealand
Sporidesmin, a mycotoxin produced by the saprophytic fungus, Pithomyces chartarum, is the cause of liver and bile duct damage that leads to elevated blood phytoporphyrin concentrations and a secondary photosensitization disease known as facial eczema (FE). Facial eczema predominantly affects sheep and cattle, and is of major economic importance in New Zealand (Smith and O'hara 1978; Di Menna et al. 2009).

Cows with clinical FE show reddening, swelling, and/ or oozing and peeling of non-pigmented or sparsely haired skin, especially of the udder, teats, and escutcheon. In the early stages, behavioural observations can include 
shade-seeking, inappetence, and irritability or discomfort at milking. Besides the detrimental effects of FE on animal production and farm economics, the welfare of many photosensitized animals can be severely compromised (Morris et al. 2004).

Despite a large amount of FE research since the first report in sheep in 1897 (Lancashire and Keogh 1968) comparatively little has focused on the disease in cattle. Publications have concentrated on case reports from natural challenges, and on the genetic susceptibility of Friesian and Jersey cattle (Morris et al. 1991a, b, 1998, 2013). Furthermore, no data has been published on FE research using the modern techniques of metabolomics.

It has been estimated that for every animal that shows clinical signs, there are 10 that are subclinically affected (Morris 2011). Subclinically affected animals show no skin abnormalities, but liver damage can be revealed by blood biochemistry tests. Measurement of the serum activity of $\gamma$-glutamyltransferase (GGT) and glutamate dehydrogenase (GDH), enzymes which indicate bile duct and liver cell damage, respectively, have been used for many years to identify subclinical and clinical cases of FE (Morris et al. 2004, 2013; Phua et al. 2009). The activity of GGT, however, shows a lag phase of 10-14 days, before increasing (Bennison et al. 2010; Di Menna et al. 2009; Morris et al. 1998; Smith and Gravett 1986). An elevation of GGT activity may be relatively minor in subclinical cases, while animals that demonstrate elevations of a thousand units (IU/L) or more generally develop photosensitization (clinical FE). Although serum GGT testing is cheap and easy to perform, the interpretation of raised activities can provide false positives for FE, since other diseases, such as photosensitisation occurring on Brassica crops can cause similar enzyme elevations, clinical signs, and liver lesions (Collett 2014; Collett and Matthews 2014). Being able to identify whether a photosensitisation is caused by sporidesmin, or some other 'toxin', will allow for specific prevention, treatment and management methods, such as removing animals from toxic pastures, carrying out spore counting, or zinc treatment. Additionally, identifying the cause as brassica crops rather than FE will minimise additional environment stress caused by unneeded addition of toxic zinc treatments.

The response lag phase, and the lack of specificity, means that elevated GGT activity has limitations as a marker of sporidesmin toxicity.

With the aim to improve the diagnostic specificity of subclinical and clinical FE in cattle, we chose to apply modern analytical technologies [mass spectrometry (MS) and nuclear magnetic resonance (NMR)] in an attempt to discover one or more serum metabolite(s) that could be a diagnostic biomarker of sporidesmin exposure. Serum samples from cows that were non-responsive, that developed subclinical disease (as indicated by slight to moderate elevations in GGT activities), or that developed clinical photosensitivity (with highly elevated GGT and skin lesions), following a single dose of sporidesmin, were compared to normal, untreated control cows.

We report here the use of an untargeted approach using ultra performance liquid chromatography (UPLC) coupled to electrospray ionization MS (ESI-MS), followed by targeted analysis using a Tribrid MS, combining quadrupole, ion trap, and Orbitrap mass analyser (tandem MS or $\mathrm{MS}^{2}$ ). Such MS-based methods allow high selectivity, sensitivity and throughput, all ideally suited to the measurement of complex biological extracts (Cao et al. 2013).

In the present study, potential candidate markers, that included a combination of taurine- and glycine-conjugated secondary bile acids (BAs) were tentatively identified by accurate mass and by consulting online databases, such as the Human Metabolome Database (HMDB) (Wishart et al. 2013) and ChemSpider (Chemspider 2015), along with $\mathrm{MS}^{2}$ fragmentation data.

\section{Experimental}

\subsection{Animals, dosing, and sample collection}

All samples for this analysis were collected during a 59-day sporidesmin dosing trial carried out at Massey University, Palmerston North, New Zealand. All cows were subjected to a 14-day baseline control sampling period (Days -14 to -1 ) and 45 days of toxin response testing (Days 0-44). The study was approved by the Massey University Animal Ethics Committee (Protocol No. 11/23).

The sporidesmin was a crude extract obtained from spores of P. chartarum dissolved in $96 \%$ ethanol, prepared at AgResearch, Ruakura, Hamilton, New Zealand. Ethanol is the solvent routinely used in sporidesmin dosing studies (Amyes and Hawkes 2014). Seventeen of twenty adult Friesian and Friesian $x$ Jersey, non-pregnant and lactating dairy cows, of mixed age, were treated with a sporidesmin dose $(0.24 \mathrm{mg} / \mathrm{kg}$; diluted with Milli-Q water) via intra-ruminal intubation, followed by $1 \mathrm{~L}$ of water. The remaining three cows served as controls and were subjected to the same regimen, excepting the sporidesmin dose was replaced by the equivalent volume of ethanol in water. Doses per cow, based on weight, are given in "Supplementary extended Appendix 1."

Blood samples were collected into vacutainer tubes (no anticoagulant) every Monday, Wednesday, and Friday morning ( $n=25$ days). Two cows (239 and 393) were humanely killed before the trial was terminated due to the development of severe clinical FE, based on humane points for ethics, therefore provided only $n=15$ samples/ 
cow. All other cows had a total of $n=25$ blood samples collected, per cow, for analytical chemistry analysis (total $n=480$ ).

Once collected, blood samples were left to stand for ca. $30 \mathrm{~min}$, and then centrifuged ( $3000 \mathrm{rpm}, 6 \mathrm{~min})$. The serum was transferred to cryovials and stored at $-80{ }^{\circ} \mathrm{C}$ pending analysis. An additional sample from each cow, collected every Monday, was submitted to New Zealand Veterinary Pathology (NZVP), Palmerston North, for serum biochemistry analysis $(n=172)$.

At the end of the sampling period, the treated cows were assigned to three groups based on their liver enzyme activities and photosensitisation observations. These groups were: non-responders (GGT activities within the reference range 0-36 IU/L for the duration of the trial), subclinical (elevated GGT activities, with no photosensitisation), and clinical (elevated GGT activities and photosensitisation).

\subsection{Liquid chromatography-mass spectrometry}

Following mixing, $50 \mu \mathrm{L}$ aliquots of thawed serum samples were treated with $220 \mu \mathrm{L}$ of ice cold acetonitrile (LC-MS grade, Thermo Fisher Scientific, Auckland, NZ), vortexed, left to stand for $15 \mathrm{~min}$ and then centrifuged $(13,000 \mathrm{rpm}, 10 \mathrm{~min})$. The supernatant was transferred to a microcentrifuge tube, and diluted with analytical grade water (Milli-Q® system, Millipore, Bedford, MA, USA), resulting in a total volume of $500 \mu \mathrm{L}$. Samples were mixed briefly (10 s vortex) and $200 \mu \mathrm{L}$ was used for analysis by UPLC coupled to a high resolution Q Exactive Orbitrap mass spectrometer (Thermo Fisher Scientific, Waltham, MA, USA).

The analysis of all samples were performed using a C18 reversed-phase column (RRHD SB-C18 column, $2.1 \mathrm{~mm} \times 150 \mathrm{~mm}, 1.8 \mu \mathrm{m}$ diameter size, Agilent, CA, USA) based on the work by Fraser et al. (2013).

The UPLC system consisted of an Accela 1250 quaternary UPLC pump, a PAL autosampler fitted with a 15,000 psi injection valve (CTC Analytics AG., Zwingen, Switzerland), a $20 \mu \mathrm{L}$ injection loop, and a Q Exactive Orbitrap mass spectrometer with electrospray ionization source (Thermo Fisher Scientific, Waltham, MA, USA). Please refer to 'Supplementary experimental extended' and Fraser et al. (2013) for detailed information on UPLC-MS experimental conditions.

Quality control samples, derived from a pooled $10 \mu \mathrm{L}$ aliquot of all samples collected during the trial, were injected at the beginning, as every 10th injection thereafter, and at the end of each batch run. These control samples were formed to monitor systematic variations in run order and batch effects. A total of 690 samples were analysed in 7 batches.

\subsection{Tandem mass spectrometry coupled to liquid chromatography}

Pooled serum samples were prepared by combining $100 \mu \mathrm{L}$ of serum from each of three clinical cows (collected on Day 16 post-dosing). $1.2 \mathrm{~mL}$ acetonitrile was added to the combined sera, and vortexed. The solution was then centrifuged $\left(12,000 \mathrm{rpm}, 10 \mathrm{~min}, 4^{\circ} \mathrm{C}\right)$. The supernatant was collected and dried in a centrifugal evaporator (Savant SC210A SpeedVac, Thermo Scientific). The dried residue was reconstituted in $100 \mu \mathrm{L}$ of 50:50 (v/v) acetonitrile:water containing $0.1 \%$ formic acid.

The serum samples were analysed using a Dionex Ultimate 3000 UPLC system (Thermo Scientific), consisting of a pump, a column compartment (set to $30{ }^{\circ} \mathrm{C}$ ), and an auto sampler (held at $10{ }^{\circ} \mathrm{C}$ ). The chromatographic separation was carried out using two identical Acquity UPLC high strength silica (HSS) T3 columns $(2.1 \mathrm{~mm} \times 100 \mathrm{~mm}$, particle size of $1.8 \mu \mathrm{m}$; Waters Corporation, Milford, MA, USA) connected in series to enhance separation.

An Orbitrap Fusion Tribrid mass spectrometer (Thermo Scientific) was used as the mass analyser, and was operated in both negative and positive ionisation modes.

Please refer to 'Supplementary experimental extended' for details of the experimental conditions used.

\subsection{Data processing and statistical analysis}

To assess how the GGT activities changed over the trial period for each animal, and each group, a generalised additive model (GAM) (Hastie and Tibshirani 1990) was fitted to the data, using RStudio software (Version 0.97.449, RStudio, Boston, MA, USA), allowing for each group to have a separate smooth average, taking into account the initial weight of the animals. Typically, the activity of GGT following sporidesmin challenge was skewed, since individual cows, within a group, could exhibit highly variable levels of response, in which case, natural $\log (\ln )$ transformed data were used.

For the UPLC/MS, the components with a retention time (RT) before $3 \mathrm{~min}$, and after $14 \mathrm{~min}$, were considered as waste; the remaining data were extracted and aligned using in-house proprietary software developed at AgResearch, Palmerston North, NZ.

Raw data was subjected to a peak detection process using PhenoAnalyzer (SpectralWorks Ltd, Manchester, UK). The key parameter settings were: area threshold $=100,000$; peak width threshold $=0.06 \mathrm{~min}$ minimum width to $0.4 \mathrm{~min}$ maximum width; $\mathrm{m} / \mathrm{z}$ peak detection window $=10 \mathrm{ppm}$ (Fraser et al. 2014). The resulting peak-area matrix data were de-isotoped using a procedure described previously (Cao et al. 2017). Any isotopes not removed by the automated de-isotoping process were removed during data processing. 
Run-order effects within each batch were normalized using linear regression (Koulman et al. 2007) and the batch effect was corrected using a parametric empirical Bayes method (sva package, RStudio) (Johnson et al. 2007). Furthermore, the peaks still showing a significant batch effect ( $F$ test, $p$ values $<0.05$ ) were removed, as described by $\mathrm{CaO}$ et al. (2017). Following the sva correction, 42 peaks were removed from the $\mathrm{CP}$ dataset and 7 from the $\mathrm{CN}$ dataset (see 'Supplementary extended Appendix 3' for a list of removed peaks). The data were ln-transformed prior to multivariate analysis (MVA) as they did not follow a normal distribution.

Following pre-treatment, a total of 450 peaks $\left(\mathrm{m} / \mathrm{z}_{-} \mathrm{RT}\right)$ were detected within the $(-)$ ESI $(\mathrm{CN})$ chromatograms and 1278 peaks $\left(\mathrm{m} / \mathrm{z}_{-} \mathrm{RT}\right)$ in the $(+) \mathrm{ESI}(\mathrm{CP})$ chromatograms. Matrix tables were produced with the predictor variables ( $m / z_{2}$ RT values) in the $X$-block and the response variables (group, day, dosing) in the $Y$-block. The resulting matrices were then analysed using SIMCA (Version 13.0.2.0, Umetrics $\mathrm{AB}, \mathrm{Sweden}$ ) and $\mathrm{RStudio}$ statistical software.

Principal component analysis (PCA), partial least squares-discriminate analysis (PLS-DA), and orthogonal PLS-DA (OPLS-DA) were performed using SIMCA. All data were pre-treated using Pareto scaling.

PLS-DA and OPLS-DA models were produced using defined classes to aid in separation of the data based on specific observations. For example, samples were classed by those measured before dosing (Days - 14 to 0) compared to those measured after dosing (Days 7-42). All PLS-DA models were validated using permutation testing (999 permutations). Further, a reliable, validated model was considered to be one with: (a) a Variable Influence of Projection (VIPcv) score of $>1$, (b) a correlation ( $p($ corr)[1]) generally $>0.4$, and (c) loadings columns with jack-knifed confidence intervals not crossing the origin. Lists of the peaks $\left(\mathrm{m} / \mathrm{z}_{-} \mathrm{RT}\right)$ of likely candidates for the separation of classes produced from this analysis were used for data interpretation.

Time series analyses were applied to the data to explore whether any mz_RT peaks may have differed over time, and between groups over time. Time series analyses were performed using in-house routines written for RStudio. $m / z \_$RT peak intensity versus time curves were plotted for each cow. The m/z_RT peaks that differed the most between groups were identified by ranking using two techniques. The first was shrinkage discriminant analysis (SDA) which computes correlation-adjusted T-scores (CAT) between the group centroids and the pooled mean for each peak (Ahdesmaki and Strimmer 2010). The second was a permutation-based $p$ value, where the ratio of between and within group variance was computed for each peak. The groups were then permuted and the ratio recomputed. The $p$-value was then calculated as the proportion of permuted ratios that were larger than the ratio from the observed data, with a small $p$ value suggesting that the results identified in the data were unlikely to have arisen by chance. These $p$ values were not corrected for multiple testing, thus a $p$ value $<0.05$ was not regarded as statistically significant. However, ranking by $p$ value did allow the identification of peaks that were more likely to separate the groups. Full details of all of the statistical methods are given in the 'supplementary experimental extended: Methods.'

Once important $m / z_{-}$RT pairs were identified for both the MVA and the time series models, the raw spectra were examined to determine whether these variables related to 'true' peaks, rather than noise, whether they were well resolved, and had high signal to noise ratios $(\mathrm{S} / \mathrm{N}>3)$. Once the peaks fulfilled the criteria, a combination of metabolite databases: the HMDB (Wishart et al. 2013), ChemSpider (Chemspider 2015), published data (Allen et al. 2011; Griffiths and Sjovall 2010), and $\mathrm{MS}^{2}$ fragmentations were used to tentatively identify metabolites.

\section{Results}

\subsection{Serum biochemistry}

Of the 17 treated cows, four were classified as non-responders, six subclinical, and seven as clinical (Table 1). The first clinical cases $(n=2)$ occurred on Day 7 and Day 9 following sporidesmin dosing.

There was no correlation between the time when clinical photosensitivity became evident and the maximum elevation of GGT. For example, one cow had a GGT activity of 863 IU/L three days prior, while a second cow had a GGT of $116 \mathrm{IU} / \mathrm{L}$ two days prior to the signs emerging.

In Fig. 1 changes in GGT activities for both the individual cows as well as the average within the groups, across time, are shown. The GGT activities remained well within the normal range for controls and non-responders, while they increased between Days 7 and 14 for subclinical animals, followed by recovery after around Day 21 . No differences were identified between control cows and non-responders over the trial period. However, clinical animals that had elevated levels through the entire trial exhibit an increase in liver enzyme activities well above normal, following dosing (Day $0)$. There is a clear difference between groups $(\mathrm{p}<0.0001)$ when compared to a baseline model with a common trend for all groups and this is primarily due to the clinical group differing (Fig. 1) from other groups. Within the clinical cow group, cows 282 and 393 exhibited more severe responses to the sporidesmin dosing than all other cows. To ensure these cows did not have a strong effect on the mean-based measures used in GAM, samples from cows 282 and 393 were removed and the models re-run (See supplementary extended Appendix 2 for plots produced from these models). When this model was compared to the original model 
Table 1 Categorization of groups based on elevated liver enzyme activities following sporidesmin dosing and visual signs of photosensitivity

\begin{tabular}{|c|c|c|c|c|c|}
\hline Category & $\mathrm{n}$ & Cow ID & $\begin{array}{l}\text { GGT* } \\
\left.\text { (IU/L } 37^{\circ} \mathrm{C}\right)\end{array}$ & $\begin{array}{l}\mathrm{GDH}^{* *} \\
\left(\mathrm{IU} / \mathrm{L} 37^{\circ} \mathrm{C}\right)\end{array}$ & Clinical signs \\
\hline \multicolumn{6}{|l|}{ Normal } \\
\hline Control & 3 & $22,152,384$ & $\begin{array}{l}21 \\
(16-27)\end{array}$ & $\begin{array}{l}15 \\
(7-59)\end{array}$ & None \\
\hline Non-responder & 4 & $195,244,374,448$ & $\begin{array}{l}21 \\
(13-33)\end{array}$ & $\begin{array}{l}18 \\
(3-65)\end{array}$ & None \\
\hline \multicolumn{6}{|l|}{ Responders } \\
\hline Subclinical & 6 & $64,222,312,395,420,424$ & $\begin{array}{l}56 \\
(15-288)\end{array}$ & $\begin{array}{l}44 \\
(6-389)\end{array}$ & None \\
\hline Clinical & 7 & $239,282,298,317,393,440,450$ & $\begin{array}{l}1102 \\
(116-2377)\end{array}$ & $\begin{array}{l}440 \\
(11-2420)\end{array}$ & $\begin{array}{l}\text { Abnormal irritability, shade seeking, increased sensitiv- } \\
\text { ity, reddening of non-pigmented skin, peeling of } \\
\text { skin*** }\end{array}$ \\
\hline
\end{tabular}

Measured GGT and GDH activities are presented as median (range) for the group from the trial, after Day 0

* $\gamma$-Glutamyl transferase; normal range is $0-36 \mathrm{UI} / \mathrm{L}$ at $37^{\circ} \mathrm{C}$ for New Zealand Veterinary Pathology laboratories

** Glutamate dehydrogenase; normal range is $8-41 \mathrm{UI} / \mathrm{L}$ at $37^{\circ} \mathrm{C}$ for New Zealand Veterinary Pathology laboratories

*** The first clinical signs appeared at Day 7, with 393 having increased sensitivity and irritability in the milking shed. The remaining cows began to show signs around Day 9 (shade seeking and/or reddening of teats)

Fig. $1 \gamma$-Glutamyltransferase (GGT) activities for each of the four defined groups, with within group averages fit, calculated using a generalised additive model. $\log _{\mathrm{e}}$ transformed data was used. Day 0 refers to the day of dosing. Shaded areas represent the $95 \%$ confidence bands for that group

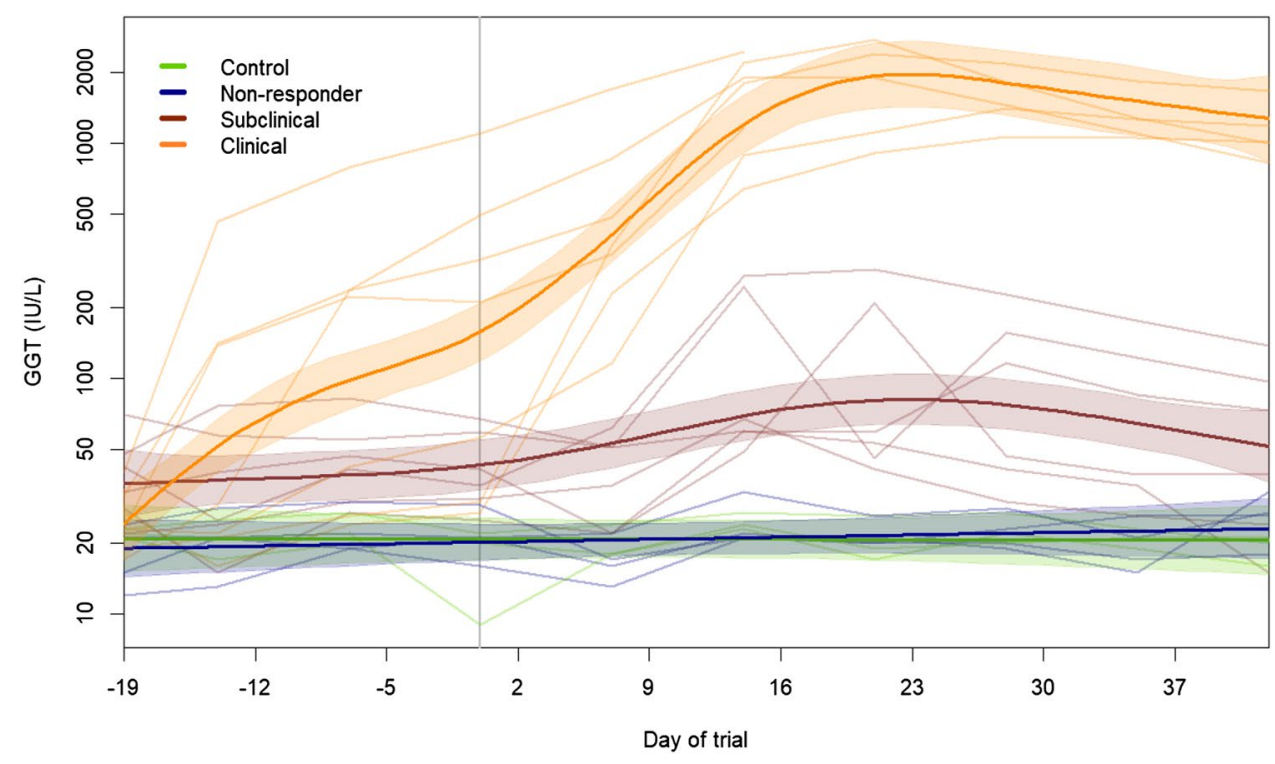

with all 20 cows, the results remained the same $(\mathrm{p}<0.0001)$ (Figures a and b in Supplementary extended Appendix 2) therefore all cows were retained in the dataset.

\subsection{Multivariate analysis}

Both the $\mathrm{CN}$ and $\mathrm{CP}$ datasets showed significant differences ( $\mathrm{CN} p=1.88 \times 10^{-28}$, CP $p=4.63 \times 10^{-9}$, OPLS-DA) between clinical cattle after dosing, and all other animal groups (Figs. 2,3), with the clinical cattle moving away from the other cow groups following a trend with time after sporidesmin dosing, and returning towards the other groups in the later weeks of the trial, consistent with a recovery phase.
The PLS-DA models were validated, with a difference between the goodness of fit $\left(\mathrm{R}^{2} 0.298\right.$ and 0.335$)$ and the predictive ability $\left(\mathrm{Q}^{2} 0.201\right.$ and 0.249$)$ of 0.097 and 0.086 for the $\mathrm{CP}$ and $\mathrm{CN}$ datasets, respectively. The $\mathrm{R}^{2} / \mathrm{Q}^{2}$ values were themselves low, and not considered reliable indicators by SIMCA definition; however, because of the inherent variable nature of large metabolomic datasets of biological origin, and the closeness of these two attributes to each other, these models were accepted (Worley and Powers 2013). In the scores plots (Figs. 2a, 3a), those samples sitting outside the grey confidence ellipse $\left(\mathrm{T}^{2}>95 \%\right)$, were not considered to be outliers, as they related to weeks 4,5 , and 6 of the trial (Days 7, 9, 11, 14, 16, and 21) — the earlier days representing 

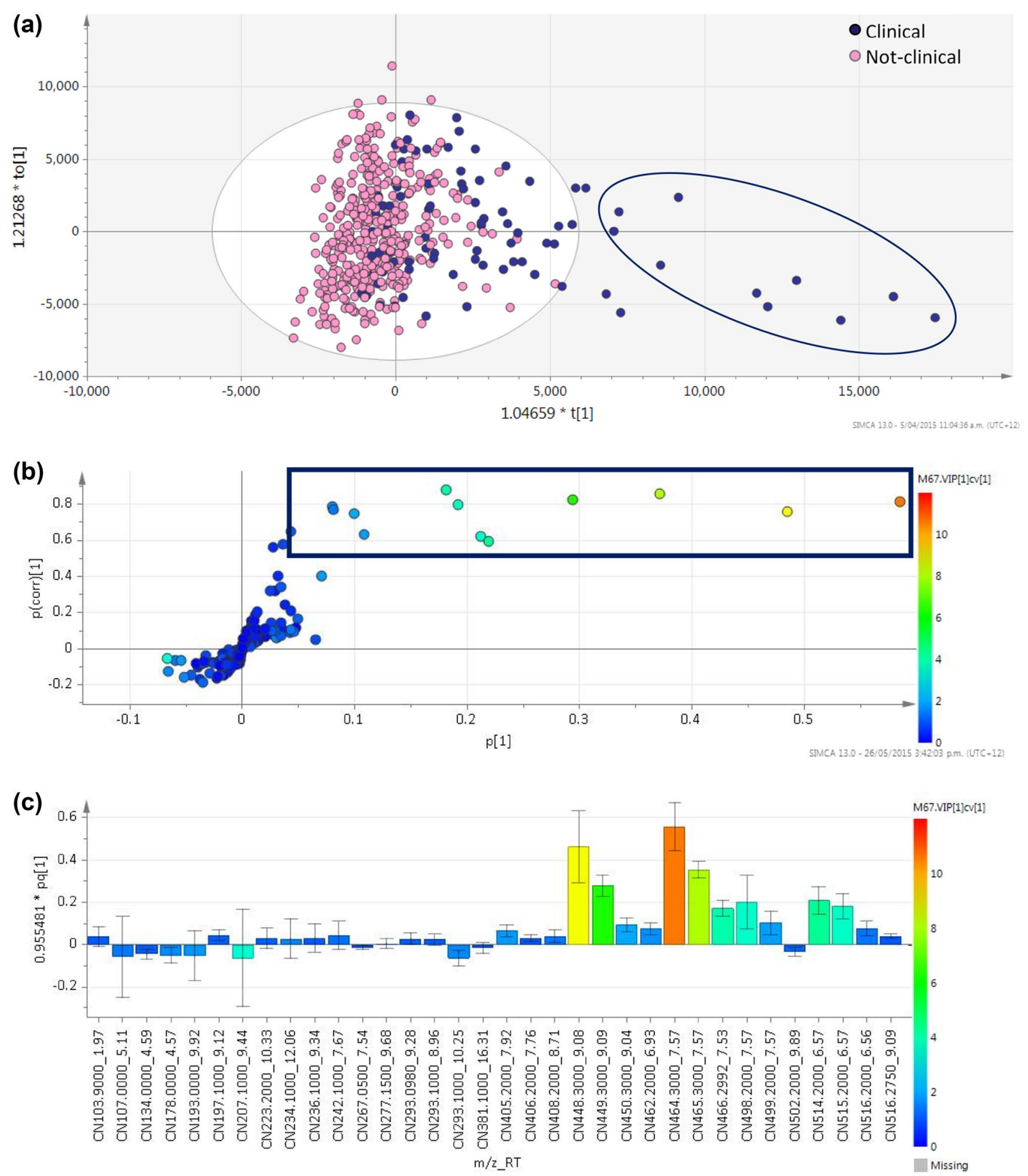

Fig. $2 \mathrm{CN}$ derived data showing orthogonal partial least squaresdiscriminant analysis (OPLS-DA) classed by clinical cows versus all other cows, using Pareto scaling; a Scores plot coloured by classes. Cow 282 clustering is shown by the ellipse; b S-plot, coloured by
Variable Influence of Projection (VIPcv) (box $=\mathrm{p}($ corr $)[1]>0.5$; VIPcv $>1$ ); and c loadings column plot showing all those with a VIPcV $>1$ 

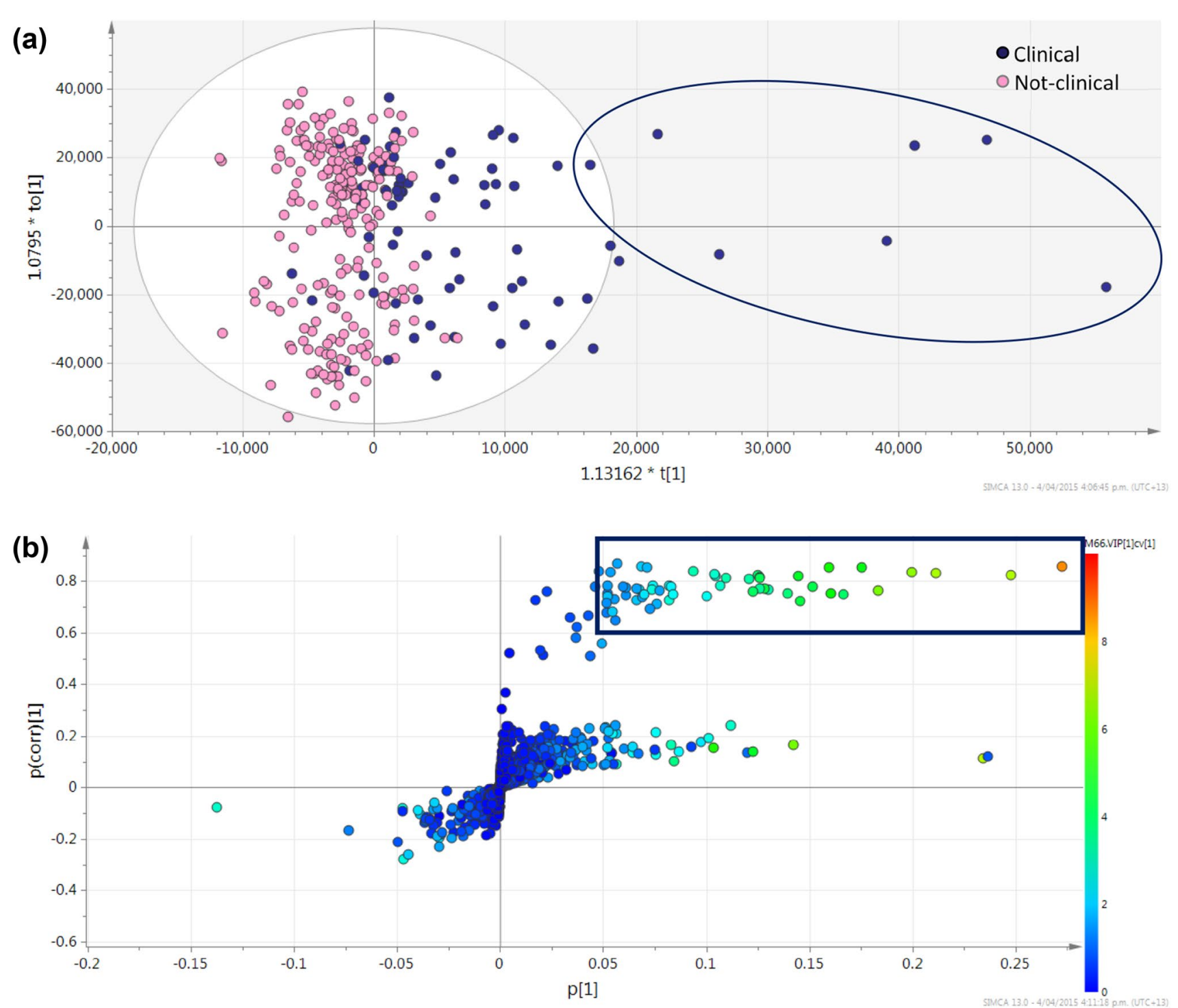

(c)

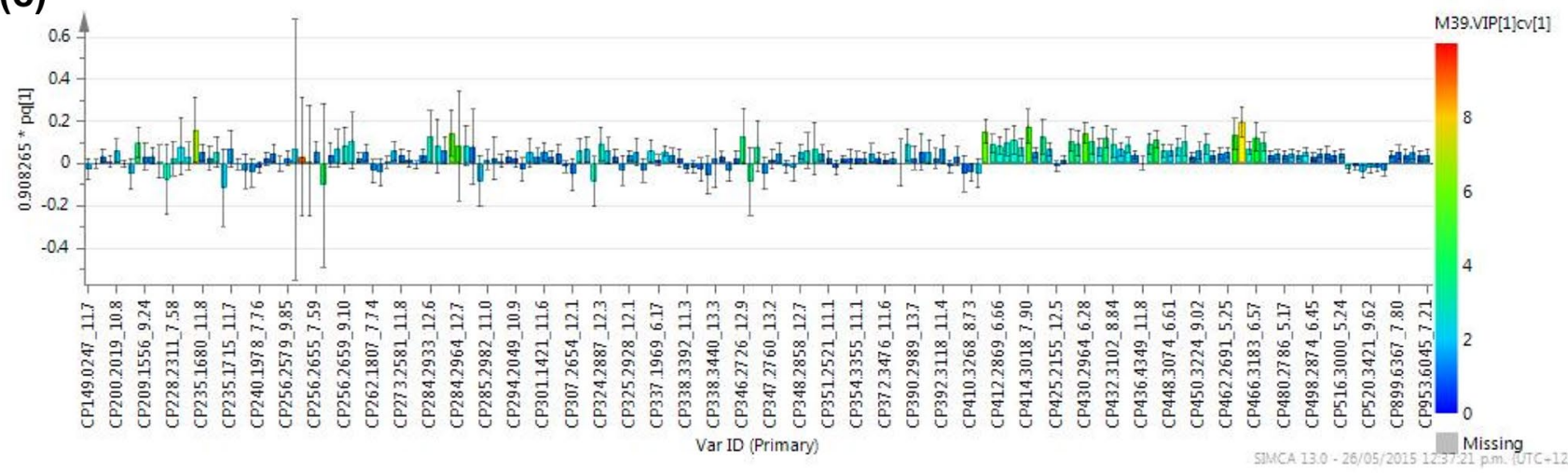

Fig. 3 CP derived data, showing orthogonal partial least squares discriminant analysis (OPLS-DA), classed by the clinical cow group, after dosing, versus all other cows and control days, using Pareto scaling; a scores plot, coloured by classes. Cow 282 clustering is rep- resented by the ellipse; $\mathbf{b}$ S-plot, coloured by Variable influence of Projection (VIPcv) (box shows p(corr)[1] $>0.5$ and VIPcv $>1$ ); and c loadings column plot showing all $\mathrm{m} / \mathrm{z}$ with a VIPcv $>1$ 
a change from 'normal' after dosing, and the latter (being closer to zero on the $x$ axis), representing a return to likeness of the other samples. Furthermore, most of the individual clinical cows appeared to cluster together, whereas cow 282, a clinical cow, differentiated the most from all, and was seen to orientate in the bottom right of the OPLS-DA plots (blue ellipse, Figs. 2a, 3a). Cow 282 was one of the more severely affected cows in the trial. In addition, these were the weeks where all of the clinical cattle had their highest GGT activity and began showing clinical signs.

The S-plot combined with VIPcv colouring (VIPcv > 1) and jack-knifed confidence levels $(>0)$ indicated 22 peaks and $76 \mathrm{~m} / \mathrm{z}_{-}$RT peaks which were relevant to class separation from the $\mathrm{CN}$ and $\mathrm{CP}$ datasets respectively. Of the 22 peaks from the $\mathrm{CN}$ dataset, a total of 9 up-regulated peaks were identified as being reliably relevant to class separation. From these, the ions at $\mathrm{m} / \mathrm{z} 464.3017$ at $7.57 \mathrm{~min}$ and 448.3074 at 9.08 min were major contributors to the differentiation between the classes. These two ions were observed as the most important in the differentiation of clinical cows from all others after dosing, with considerably higher peak areas in the first two weeks after dosing, than in all other samples. These ions could be tentatively identified using accurate mass and the HMDB as glycocholic acid and glycochenodeoxycholic acid.

Of the 76 peaks in the CP dataset, 57 had a p(corr) [1] $>0.5$. These 57 up-regulated peaks were considered reliable for class separation. Of these, the 6 major contributors to the class separation were at $\mathrm{m} / \mathrm{z} 466.3174$ at $6.32 \mathrm{~min}$, 414.3018 at $7.90 \mathrm{~min}, 412.2866$ at $6.38 \mathrm{~min}, 430.2964$ at $6.28 \mathrm{~min}, 466.3155$ at $7.31 \mathrm{~min}$, and 448.3075 at $6.31 \mathrm{~min}$ (Supplementary extended Appendix 4).

Accurate masses were used to tentatively identify the metabolites using the HMDB. These $\mathrm{m} / \mathrm{z}$ values were consistent with a combination of BAs, such as glycocholic acid and 3-oxo-4,6, choladienoic acid, as well as amino acid derivatives, and minor metabolites of fatty acids. A number of potential isobaric isomers were also detected, including $\mathrm{m} / \mathrm{z} 337.2529$ (RT 6.31) and 337.2540 (RT 6.53) $\left([\mathrm{M}+\mathrm{H}]^{+}\right)$.

Table 2 and supplementary extended Appendix 4 summarise the peaks that were responsible for the differences between the clinical cattle compared to the other groups, as observed in the OPLS-DA models for $\mathrm{CN}$ and $\mathrm{CP}$ data, respectively.

No validated models could be used to separate any other combinations of the treated groups.

\subsection{Time series analysis}

Changes in the amount of certain metabolites detected in both $\mathrm{CN}$ and $\mathrm{CP}$ modes were seen between control cows and the clinical cows after dosing. The changes in the curves follow an expected shape, increasing after dosing and decreasing at the end of the trial. The exclusion of the clinical cow group revealed no obvious differences between the remaining groups (controls, non-responders, and subclinicals). The peaks in the raw LC-MS spectra that showed a clear visual group differentiation were examined in order to detect false positives and it was noted whether any compounds were detected in both the $\mathrm{CN}$ and $\mathrm{CP}$ ionization modes. Figure 4 shows an example of this, where corresponding $\mathrm{m} / \mathrm{z}$ values were detected.

In summary, nine and twenty-two peaks that were considered to be the main components causing the observed differences between clinical and all other cow groups were extracted from both the $\mathrm{CN}$ and the $\mathrm{CP}$ datasets (summarised in Table 2 and supplementary extended Appendix 4, respectively). Of the peaks identified as major contributors to the differentiation of clinical cows by the time series methods, five of the nine $\mathrm{CN}$ peaks and 18 of the $22 \mathrm{CP}$ peaks were also identified by OPLS-DA. This shows the complementary nature of these two techniques.

\subsection{Targeted analysis using the Orbitrap tandem mass spectrometer}

According to VIPcv values, time series combined rankings, and the examination of raw spectra, a total of nine and 22 peaks from CN and CP ESI-MS analysis, respectively, were selected for $\mathrm{MS}^{2}$ analysis (Table 2 and supplementary extended Appendix 4, respectively).

As an example from the $\mathrm{CN}$ dataset, chromatographic and $\mathrm{MS}^{2}$ data are shown in Fig. 5 for the compound eluting at $9.12 \mathrm{~min}$ in the pooled sample. For $\mathrm{m} / \mathrm{z} 464$, the product ions at $\mathrm{m} / \mathrm{z} 420.3159$ (loss of $44 \mathrm{amu}, 1 \times \mathrm{CO}_{2}$ ), 446.2954 (loss of $18 \mathrm{amu}, 1 \times \mathrm{H}_{2} \mathrm{O}$ ), and 402.3050 (loss of $62 \mathrm{amu}, \mathrm{H}_{2} \mathrm{O}+\mathrm{CO}_{2}$ ) were formed during the $\mathrm{MS}^{2}$ analysis, tentatively indicating that it is a type of organic acid.

A corresponding protonated compound $\left([\mathrm{M}+\mathrm{H}]^{+}\right)$ was detected at $\mathrm{m} / \mathrm{z} 466$ at $9.15 \mathrm{~min}$ from the CP analysis. The product ions at $\mathrm{m} / \mathrm{z} 448.3046,430.2940$, and 412.2833 were observed during the $\mathrm{MS}^{2}$ analysis. Owing to the detection from both $\mathrm{MS}$ and $\mathrm{MS}^{2}$ analysis, the ions 448.3046 (loss of $18 \mathrm{amu}, 1 \times \mathrm{H}_{2} \mathrm{O}$ ), 430.2940 (loss of 36 amu, $2 \times \mathrm{H}_{2} 0$ ), and 412.2833 (loss of $54 \mathrm{amu}, 3 \times \mathrm{H}_{2} \mathrm{O}$ ) originate from the same compound $\mathrm{m} / \mathrm{z}$ 466.3166, which readily loses water in CP-MS analysis. Neutral water loss is common for hydroxylated compounds, and the occurrence of multiple water losses lead to the conclusion that $\mathrm{m} / \mathrm{z} 466.3166$ contains three hydroxyl groups. An elemental composition of $\mathrm{C}_{26} \mathrm{H}_{44} \mathrm{NO}_{6}^{+}$(0.612 ppm error) was determined. Taking into account that the molecule contains three $\mathrm{OH}$ groups, it is most likely glycocholic acid. For the $\mathrm{m} / \mathrm{z}$ 464.3018 (RT 9.12, $[\mathrm{M}-\mathrm{H}]^{-}$) a likely elemental composition of $\mathrm{C}_{26} \mathrm{H}_{42} \mathrm{NO}_{6}{ }^{-}(-0.024 \mathrm{ppm}$ error) was also determined, and is also likely to be glycocholic acid detected 
Table 2 Summary of detected compounds from CN LC-MS and $\mathrm{MS}^{2}$ analysis of serum from the clinical cows, using MVA and time series techniques

\begin{tabular}{|c|c|c|c|c|c|c|c|}
\hline $\begin{array}{l}{[\mathrm{M}-\mathrm{H}]^{-}} \\
m / z\end{array}$ & $\begin{array}{l}\text { LC-MS } \\
\text { RT (min) }\end{array}$ & $\begin{array}{l}{[\mathrm{M}-\mathrm{H}]^{-}} \\
m / z\end{array}$ & $\begin{array}{l}\text { LC-MS/MS } \\
\text { RT (min) }\end{array}$ & $\begin{array}{l}\mathrm{MS}^{2} \text { product ions } \\
\text { (abundance) [loss (amu)] }\end{array}$ & $\begin{array}{l}\text { Elemental composi- } \\
\text { tion of the parent } \\
\text { ion }\end{array}$ & Error (ppm) & Proposed metabolite identity \\
\hline 462.28673 & 6.93 & 462.29218 & 8.53 & $\begin{array}{l}444.27602(30 \%)(-18) \\
418.29699(100 \%)(-43) \\
400.28595(20 \%)(-62)\end{array}$ & $\begin{array}{l}\mathrm{C}_{26} \mathrm{H}_{40} \mathrm{O}_{6} \mathrm{~N} \\
\mathrm{C}_{27} \mathrm{H}_{36} \mathrm{O}_{2} \mathrm{~N}_{5} \\
\mathrm{C}_{13} \mathrm{H}_{38} \mathrm{O}_{8} \mathrm{~N}_{10}\end{array}$ & $\begin{array}{l}3.646 \\
0.818 \\
-0.280\end{array}$ & Unknown \\
\hline 464.30175 & 7.57 & 464.30758 & 9.12 & $\begin{array}{l}446.29515(20 \%)(-18) \\
420.31569(20 \%)(-43) \\
402.30485(100 \%)(-62)\end{array}$ & $\mathrm{C}_{26} \mathrm{H}_{42} \mathrm{O}_{6} \mathrm{~N}$ & -0.024 & Glycocholic acid \\
\hline 464.30329 & 6.56 & 464.30692 & 9.12 & $\begin{array}{l}446.29279(20 \%)(-18) \\
420.31555(25 \%)(-44) \\
402.30515(100 \%)(-62) \\
384.29175(14 \%)(-80) \\
353.25220(5 \%)(-111) \\
400.28992(10 \%)(-64)\end{array}$ & $\mathrm{C}_{26} \mathrm{H}_{42} \mathrm{O}_{6} \mathrm{~N}$ & 3.4 & Glycocholic acid \\
\hline 516.27500 & 9.09 & 516.29710 & 10.60 & $448.30762(100 \%)(-67)$ & Unknown & - & Unknown \\
\hline 514.28452 & 6.57 & 514.29800 & 8.89 & $\begin{array}{l}496.27597(10 \%)(-18) \\
412.28754(5 \%)(-102) \\
371.26074(5 \%)(-143) \\
353.25009(8 \%)(-161)\end{array}$ & $\mathrm{C}_{26} \mathrm{H}_{45} \mathrm{O}_{7} \mathrm{NS}$ & 0.123 & Taurocholic acid \\
\hline 478.31760 & 7.57 & 478.29720 & 13.07 & $\begin{array}{l}281.25035(100 \%)(-197) \\
214.04946(2 \%)(-264) \\
196.03867(4 \%)(-282)\end{array}$ & Unknown & - & Unknown \\
\hline 448.30746 & 9.08 & 448.31360 & 10.60 & $\begin{array}{l}430.29821(15 \%)(-18) \\
404.31877(100 \%)(-44) \\
386.30826(5 \%)(-62) \\
355.26639(5 \%)(-93)\end{array}$ & $\mathrm{C}_{26} \mathrm{H}_{42} \mathrm{O}_{5} \mathrm{~N}$ & 1.368 & $\begin{array}{l}\text { Glycochenodeoxycholic acid } \\
\text { / Deoxyglycocholic acid }\end{array}$ \\
\hline 432.31274 & 10.94 & 432.31779 & 12.18 & $\begin{array}{l}388.32431(100 \%)(-44) \\
173.48961(15 \%)(-275)\end{array}$ & $\mathrm{C}_{26} \mathrm{H}_{42} \mathrm{O}_{4} \mathrm{~N}$ & 1.869 & $\begin{array}{l}\text { Lithocholic acid glycine } \\
\text { conjugate }\end{array}$ \\
\hline 498.29104 & 7.57 & 498.29105 & 10.74 & $\begin{array}{l}355.26587(100 \%)(-143) \\
414.30334(5 \%)(-84)\end{array}$ & $\mathrm{C}_{26} \mathrm{H}_{45} \mathrm{NO}_{6} \mathrm{~S}$ & 3.127 & $\begin{array}{l}\text { Taurochenodeoxycholic } \\
\text { acid/Taurodeoxycholic } \\
\text { acid }\end{array}$ \\
\hline
\end{tabular}

All components were up-regulated in comparison to the other cow groups

in negative ionization mode. Another $\mathrm{m} / \mathrm{z} 464.3033$ (RT 6.56) peak was identified as being instrumental in the difference between clinical and all other cows after dosing. With similar accurate masses and product ions, it is likely that these are isobaric isomers. Other isobaric isomers were detected, including $\mathrm{m} / \mathrm{z} 472.3031$ (RT 8.78) and 472.3039 (RT 7.66) in the CP MS spectra. The protonated compound $\left([\mathrm{M}+\mathrm{H}]^{+}\right)$detected at $\mathrm{m} / \mathrm{z} 450.3209$ at $8.52 \mathrm{~min}$ from the CP-MS analysis produced two main product ions, 432.3087 and 414.2982. The fragmentation pattern, showing a loss of one and two $\mathrm{H}_{2} \mathrm{O}$ molecules, matched with that of glycochenodeoxycholic acid $(-3.546$ and 0.228 ppm errors, respectively). Accurate mass measurements for both the precursor and product ions are summarized in Table 2 and supplementary extended Appendix 4 from the $\mathrm{CN}$ and $\mathrm{CP}$ $\mathrm{MS}^{2}$ analysis, respectively.

The most prominent changes seen between the groups, after dosing, were observable among the compound class of BAs. Distinguishing between the different BAs is often difficult, with the only differences being the presence or absence of hydroxyl groups in positions 3, 7, and 12. Tentatively, five BAs were detected that exhibited an increase in the clinical group after dosing, but not in the other groups. Glycocholic acid, taurocholic acid, taurochenodeoxycholic acid, and glycochenodeoxycholic acid (or deoxycholic acid) were detected in both $\mathrm{CP}$ and $\mathrm{CN}$ datasets, providing strong evidence for their presence in clinical cattle following liver damage caused by a sporidesmin dose. Lithocholic acid was only detected in the $\mathrm{CN}$ dataset.

\section{Discussion}

The intention of this work was to differentiate between cows responding variably to a single sporidesmin dose, using UPLC/ESI-MS and UPLC/MS ${ }^{2}$ of serum, with the aim to use individual metabolites as potential serum markers for early stage FE in lactating cows. The clinical cow group was able to be clearly differentiated using chromatography coupled to CP and CN ESI-MS. Tandem MS was able to 

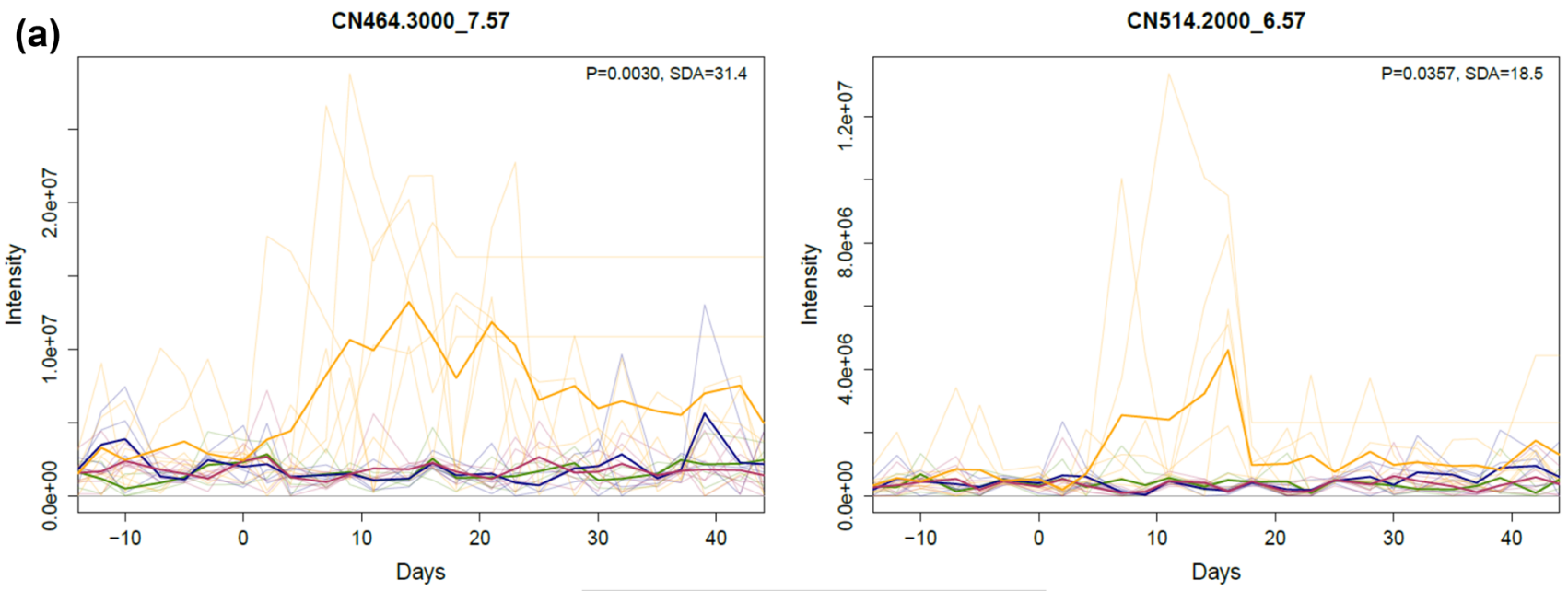

\section{- Control $\quad$ Subclinical \\ - Non-responder $=$ Clinical}

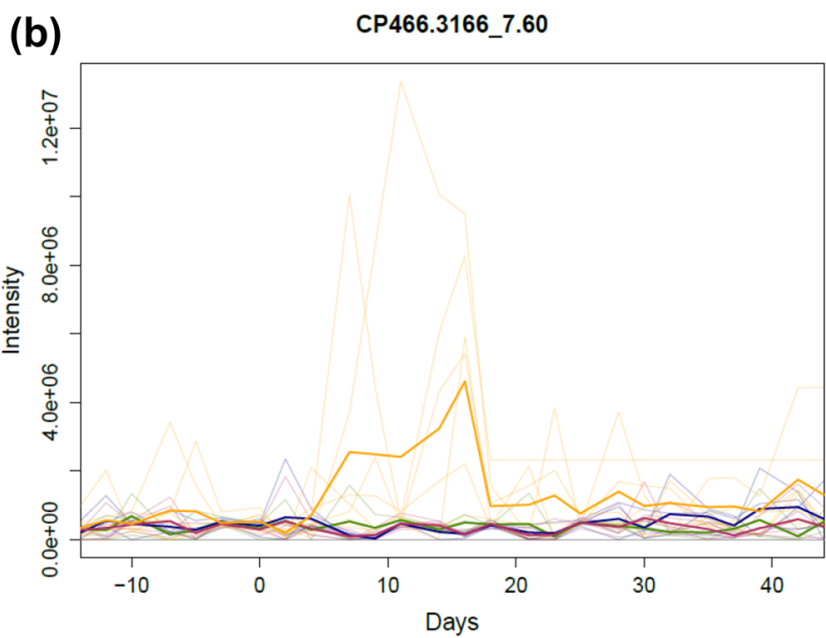

Fig. 4 Time series of the main two mz_RT peaks identified as showing a major difference in the clinical cows, only after dosing (Day $0 ; \mathrm{p}<0.05$ and true peaks), derived from a CN, and $\mathbf{b}$ CP ESI-MS analysis. The compounds were shown to increase from ca. Day 5 onwards, before any significant increases in GGT activities were

tentatively identify nine of the peaks that were relevant to clinical cows after dosing. All of the identified peaks were taurine- and glycine-conjugated secondary BAs. Bile acids are physiological detergents that facilitate excretion, absorption, and transport of fats and sterols in the intestine and liver, and are essential for the digestion and absorption of hydrophobic nutrients (Griffiths and Sjovall 2010; Chiang 2009).

For the clinical cow group, glycocholic acid, taurocholic acid, taurochenodeoxycholic acid, as well as one peak that was narrowed down to two likely BAs, glycochenodeoxycholic acid or deoxyglycocholic acid, appeared in both $\mathrm{CN}$ and CP datasets as being elevated. A lithocholic acid-glycine conjugate was identified in negative mode only. All of

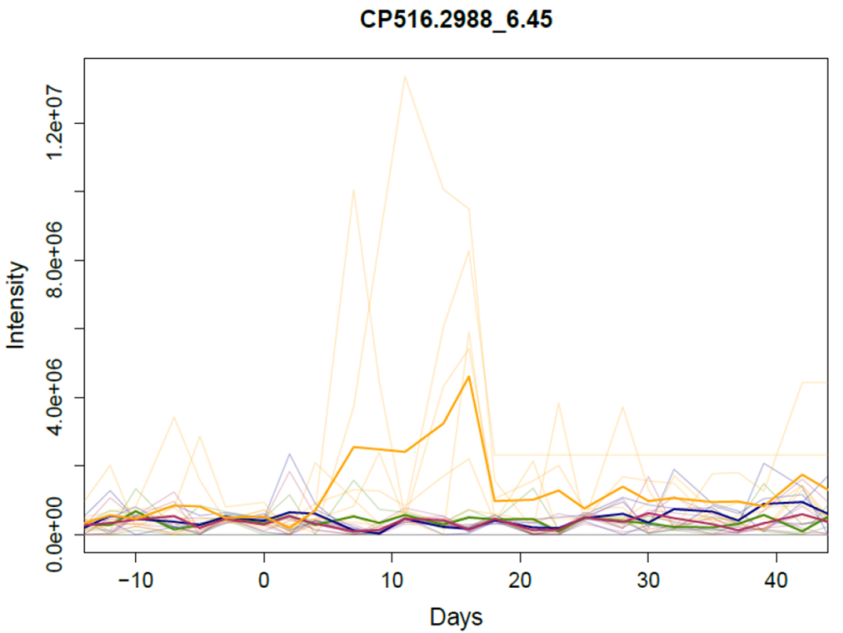

observed. The observed temporal variations, and corresponding RT and $\mathrm{m} / \mathrm{z}$ values, could be attributed to equivalent compounds, i.e. MW 465 (left) and MW 515 (right). Pale lines represent the time profiles of each m/z_RT pair for each cow. The thick lines represent the average time profiles for the groups

these metabolites were elevated in the clinical cows, when compared to the control days for the same cows, plus the control cows, and all other groups after dosing. The weeks where the concentrations of BAs were elevated in these cows correlated with the highest GGT activity and when these cows started showing clinical signs of FE. The pattern of an increase directly after toxin ingestion followed by a decrease can be expected with disease progression. This clearly correlated with the time-series analysis where an increase in BAs is observed from day 5 onwards (post-dose) then begin returning to normal towards the end of the trial..

Three of the seven cows categorised as clinical, based on liver enzyme activities and clinical observations, showed elevations in GGT activities before the dose of sporidesmin 


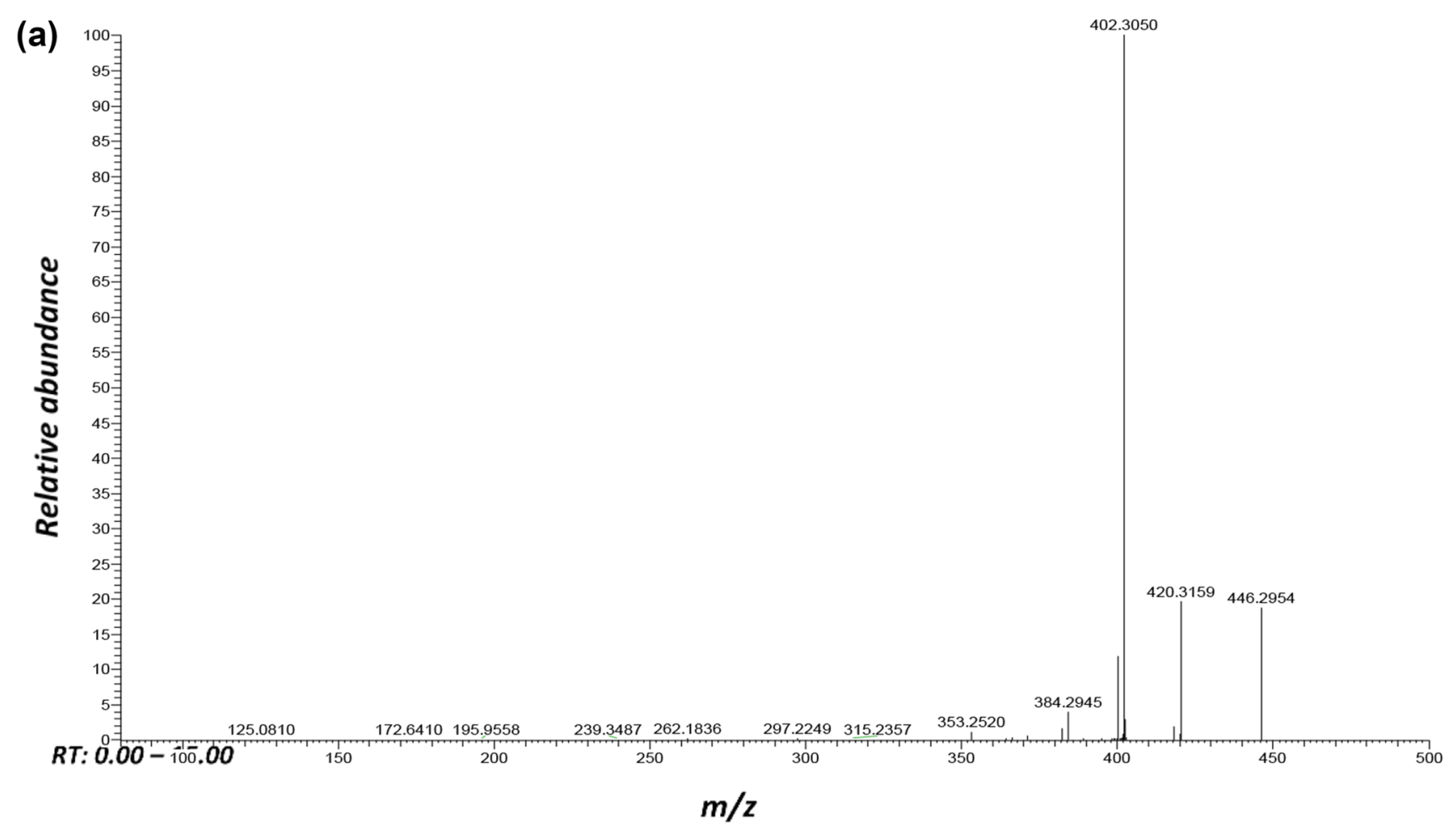

(b) 100

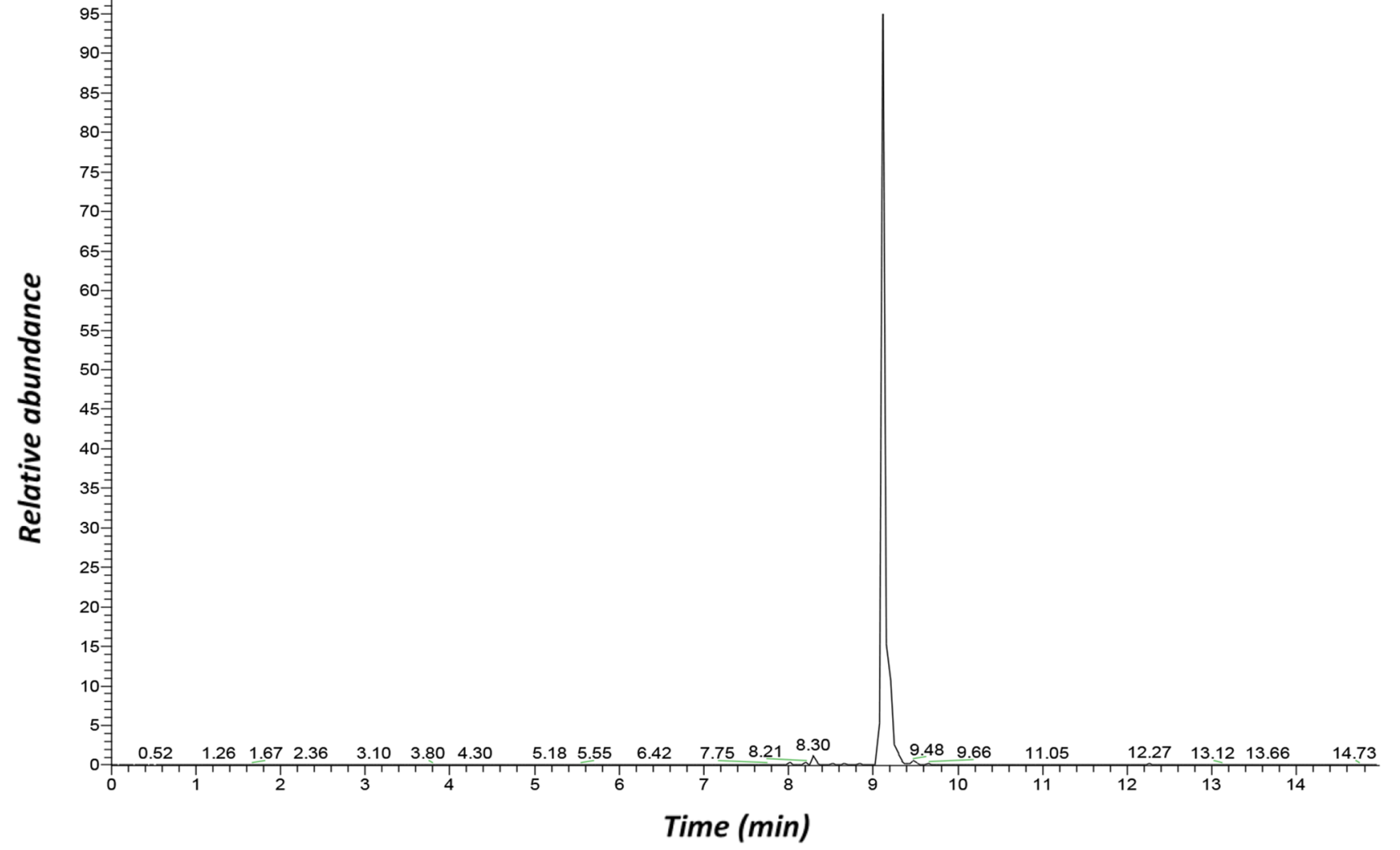

Fig. 5 Liquid chromatography negative electrospray ionisation tandem mass spectrometry analysis of serum, showing the m/z 464 MS2 data (a) eluting at $9.12 \mathrm{~min}$ (b) 
was administered on Day 0. The reason for this is unknown. The cows originated from the same farm which had no history of FE, and prior to purchase the GGT activities were all within normal reference range. On arrival at Massey, all cows were grazed on paddocks that had been sprayed with a fungicide and $P$. charatarum spore counts in pasture and faeces had been assessed before and during the trial. The three control cows were randomly selected, and all cows received the same dose for their body weight. In addition, the livers of all cows were examined grossly and histologically, and none showed any evidence of chronic (i.e. preexisting) sporidesmin damage. Therefore, we concluded that the elevations in enzyme activities before dosing was not related to sporidesmin exposure at the farm of origin or at Massey University.

In addition, although there are other mycotoxins which are commonly present in pastures around the same time as sporidesmin, such as ryegrass and fescue endophytes (Nicol and Klotz 2016; Rogers et al. 2016), the compounds produced by these endophytes do not cause liver damage (Rogers et al. 2016; Nicol and Klotz 2016; Di Menna et al. 2012). Aflatoxin, which is also present when humid conditions develop, does cause liver damage, however is a storage mycotoxin and therefore present in post-harvest stored grains nuts (Keyl and Booth 1971), which the cattle in this trial had no access to. The liver lesions cause by aflatoxin, are distinctly different to those caused by sporidesmin (Cullen and Stalker 2016), and were not detected during histological analysis.

It is well established that concentrations of BAs increase in the liver and blood during cholestasis due to leakage of bile into the parenchyma of the liver (Woolbright et al. 2014; Zhang et al. 2012; Fickert et al. 2002). It is often assumed that the increases in cytotoxic BAs are the cause of, or at least involved in, the progressive hepatocellular injury that occurs during cholestasis. Zhang et al. (2012) showed that the concentration of BAs was elevated in the serum after bile duct ligation in rats. Chenodeoxycholate is known to be directly cytotoxic for hepatocytes (Miyazaki et al. 1984). In addition, lithocholic acid, a bacterial metabolite of chenodeoxycholate produced in the intestinal tract, has been implicated as a possible hepatotoxin (Miyazaki et al. 1984). Glycochenodeoxycholic acid is the most toxic known form of chenodeoxycholate, and has also been shown to induce hepatocellular injury in a dose-dependent manner in human patients (Spivey et al. 1993; Trottier et al. 2012; Woolbright et al. 2015; Siviero et al. 2008).

Most studies focus on glycochenodeoxycholic acid and taurocholic acid, with some mention of taurochenodeoxycholic acid, glycocholic acid, lithocholic acid, and deoxyglycocholic acid. One study showed that $95 \%$ of biliary BAs in bovids was made up of three types: cholic acid, chenodeoxycholic acid and deoxycholic acid (Hagey et al.
1997). It has been suggested that glycine-conjugated BAs are more cytotoxic than those that are taurine-conjugated; however this may be due to the greater prevalence of glycine-conjugated BAs (Woolbright et al. 2015). Both glycine- and taurine-conjugated BAs were identified in the serum of clinical cows in the present study.

Other recent studies have suggested that BAs are not directly hepatotoxic, but instead increase the expression of proinflammatory mediators, such as cytokines, that promote hepatic inflammation and significant liver injury through the recruitment of neutrophils and macrophages (Allen et al. 2011; Woolbright et al. 2015; Zhang et al. 2012).

Irrespective of the mechanism, it appears that the increase in the concentration of various serum BAs seen in cows that became clinically affected, occurs following primary damage to bile ducts. Sporidesmin causes a necrotising cholangitis (Hohenboken et al. 2000), which manifests as cholestasis in severe cases. In addition to cholestasis, sporidesmin has been shown to inhibit BA uptake into hepatocytes (Cordiner and Jordan 1983). Furthermore, previous work by Gallagher (1964) and Middleton (1974) demonstrated sporidesmininduced mitochondrial swelling, suggesting that sporidesmin acts on hepatocyte membranes, and may increase permeability, and efflux of BAs from the cytoplasm.

In conclusion, the use of MS provided additional data concerning the latter stages of clinical FE in dairy cattle. Sporidesmin damage, at the dose given in this study, appeared to manifest itself quickly in those cows that were more susceptible to the toxin. It is hypothesised that any metabolites produced directly from sporidesmin, and/or other compounds, during the initial attack on the bile ducts would have been removed from the blood before the first blood samples were taken, $48 \mathrm{~h}$ after dosing. The results presented here could be used as a basis for more targeted analysis in the immediate aftermath of dosing, better processing, refinement of equipment parameters, accurate quantification, and identification of marker metabolites. To our knowledge this is the first time an elevation of deoxyglycholic acid has been detected in association with bovine FE. Future studies should investigate the BAs as a target set of biomarkers for liver damage caused by sporidesmin intoxication.

Acknowledgements This research was primarily funded by MBIE grants. We appreciate additional funding from Massey University, University of Auckland and the Sinclair Cummings scholarship. We acknowledge the help of the Massey University Dairy No. 4 farm staff, NZVP staff, Mike Hogan (Massey University post mortem facility), Silver Fern Farms Pacific (Hastings), AgResearch staff (Palmerston North), and Neville Amyes (AgResearch Ruakura) for his advice and provision of sporidesmin.

\section{Compliance with ethical standards}

Conflict of interest The authors declare no conflicts of interest. 
Research involving animal and human rights All experimental procedures were approved by the Massey University Animal Ethics Committee (MUAEC; Protocol No. 11/23), and animals were cared for in accordance with the New Zealand Animal Welfare Act (1999).

Open Access This article is distributed under the terms of the Creative Commons Attribution 4.0 International License (http://creativeco mmons.org/licenses/by/4.0/), which permits unrestricted use, distribution, and reproduction in any medium, provided you give appropriate credit to the original author(s) and the source, provide a link to the Creative Commons license, and indicate if changes were made.

\section{References}

Ahdesmaki, A., \& Strimmer, K. (2010). Feature selection in omics prediction problems using CAT scores and false non-discovery rate control. Annals of Applied Statistics, 4, 503-519. Preprint available from http://arxiv.org/abs/0903.2003.

Allen, K., Jaeschke, H., \& Copple, B. L. (2011). Bile acids induce inflammatory genes in hepatocytes: A novel mechanism of inflammation during obstructive cholestasis. American Journal of Pathology, 178, 175-186.

Amyes, N. C., \& Hawkes, A. D. 2014. Ramguard-Increasing the Tolerance to Facial Eczema in New Zealand Sheep. In Proceedings of the New Zealand Society of Animal Production, Napier.

Bennison, J. J., Nottingham, R. M., Key, E. L., \& Parkins, J. J. (2010). The effect of zinc oxide and elemental zinc boluses on the concentrations of $\mathrm{Zn}$ in serum and faeces, and on providing protection from natural Pithomyces chartarum challenge in calves. New Zealand Veterinary Journal, 58, 196-200.

Cao, M., Fraser, K., Jones, C., Stewart, A., Lyons, T., Faville, M., \& Barrett, B. (2017). Untargeted metabotyping Lolium perenne reveals population-level variation in plant flavonoids and alkaloids. Frontiers in Plant Science, 8, 1-12.

Cao, M., Fraser, K., \& Rasmussen, S. (2013). Computational analyses of spectral trees from electrospray multi-stage mass spectrometry to aid metabolite identification. Metabolites, 3, 1036-1050.

Chemspider, A. 2015. ChemSpider Home Page [Online]. Available http://www.chemspider.com/, Accessed April 2015.

Chiang, J. Y. L. (2009). Bile acids: Regulation of synthesis. Journal of Lipid Research, 50, 1955-1966.

Collett, M. G. (2014). Bile duct lesions associated with turnip (Brassica rapa) photosensitization compared with those due to sporidesmin toxicosis in dairy cows. Veterinary Pathology, 51, 986-991.

Collett, M. G., \& Matthews, Z. M. (2014). Photosensitivity in cattle grazing Brassica crops. International Journal of Poisonous Plant Research, 3,, 6-21.

Cordiner, S. J., \& Jordan, T. W. (1983). Inhibition by sporidesmin of hepatocyte bile-acid transport. Biochemical Journal, 212, 197-204.

Cullen, J. M., \& Stalker, M. J. (2016). Liver and biliary system. In M. G. Maxie (Ed.), Jubb, Kennedy, and Palmer's pathology of domestic animals (6th edn.). St. Louis, MI: Elsevier.

Di Menna, M. E., Finch, S. C., Popay, A. J., \& Smith, B. L. (2012). A review of the Neotyphodium lolii/Lolium perenne symbiosis and its associated effects on animal and plant health, with particular emphasis on ryegrass staggers. New Zealand Veterinary Journal, $60,315-328$.

Di Menna, M. E., Smith, B. L., \& Miles, C. O. (2009). A history of facial eczema (pithomycotoxicosis) research. New Zealand Journal of Agricultural Research, 52, 345-376.
Fickert, P., Zollner, G., Fuchsbichler, A., Stumptner, C., Weiglein, A. H., Lammert, F., Marschall, H. U., Tsybrovskyy, O., Zatloukal, K., Denk, H., \& Trauner, M. (2002). Ursodeoxycholic acid aggravates bile infarcts in bile duct-ligated and Mdr2 knockout mice via disruption of cholangioles. Gastroenterology, 123, 1238-1251.

Fraser, K., Lane, G. A., Otter, D. E., Harrison, S. J., Quek, S. Y., Hemar, Y., \& Rasmussen, S. (2014). Non-targeted analysis by LC-MS of major metabolite changes during the oolong tea manufacturing in New Zealand. Food Chemistry, 151, 394-403.

Fraser, K., Lane, G. A., Otter, D. E., Hemar, Y., Quek, S. Y., Harrison, S. J., \& Rasmussen, S. (2013). Analysis of metabolic markers of tea origin by UHPLC and high resolution mass spectrometry. Food Research International, 53, 827-835.

Gallagher, C. H. (1964). Effect of sporidesmin on liver enzyme systems. Biochemical Pharmacology, 13, 1017-1026.

Griffiths, W. J., \& Sjovall, J. (2010). Bile acids: Analysis in biological fluids and tissues. Journal of Lipid Research, 51, 23-41.

Hagey, L. R., Gavrilkina, M. A., \& Hofmann, A. F. (1997). Agerelated changes in the biliary bile acid composition of bovids. Canadian Journal of Zoology-Revue Canadienne De Zoologie, $75,1193-1201$.

Hastie, T. J., \& Tibshirani, R. J. (1990). Generalized Additive Models. Florida: Chapman \& Hall/CRC.

Hohenboken, W. D., Robertson, J. L., Blodgett, D. J., Morris, C. A., \& Towers, N. R. (2000). Sporidesmin-induced mortality and histological lesions in mouse lines divergently selected for response to toxins in endophyte-infected fescue. Journal of Animal Science, $78,2157-2163$.

Johnson, W. E., Li, C., \& Rabinovic, A. (2007). Adjusting batch effects in microarray expression data using empirical Bayes methods. Biostatistics, 8, 118-127.

Keyl, A. C., \& Booth, A. N. (1971). Aflatoxin effects in livestock. Journal of the American Oil Chemists Society, 48, 599-\&.

Koulman, A., Tapper, B. A., Fraser, K., Cao, M. S., Lane, G. A., \& Rasmussen, S. (2007). High-throughput direct-infusion ion trap mass spectrometry: A new method for metabolomics. Rapid Communications in Mass Spectrometry, 21, 421-428.

Lancashire, J. L., \& Keogh, R. G. (1968). Facial eczema and grazing management. In Massey Sheepfarming Annual, pp. 29-33.

Middleton, M. C. (1974). Effects of mycotoxin sporidesmin on swelling and respiration of liver-mitochondria. Biochemical Pharmacology, 23, 801-810.

Miyazaki, K., Nakayama, F., \& Koga, A. (1984). Effect of chenodeoxycholic acid and ursodeoxycholic acids on isolated adult human hepatocytes. Digestive Diseases and Sciences, 29, 1123-1130.

Morris, C. A. (2011). Managing increasing facial eczema challenges in dairying. Sustainable Farming Fund project report.

Morris, C. A., Burton, L. J., Towers, N. R., Cullen, N. G., Rendel, J. M., \& Johnson, D. L. (1998). Genetics of susceptibility to facial eczema in Friesian and Jersey cattle. New Zealand Journal of Agricultural Research, 41, 347-357.

Morris, C. A., Phua, S. H., Cullen, N. G., \& Towers, N. R. (2013). Review of genetic studies of susceptibility to facial eczema in sheep and dairy cattle. New Zealand Journal of Agricultural Research, 56, 156-170.

Morris, C. A., Towers, N. R., Hohenboken, W. D., Maqbool, N., Smith, B. L., \& Phua, S. H. (2004). Inheritance of resistance to facial eczema: A review of research findings from sheep and cattle in New Zealand. New Zealand Veterinary Journal, 52,, 205-215.

Morris, C. A., Towers, N. R., Smith, B. L., \& Southey, B. R. (1991a). Progeny testing bulls for susceptibility to facial eczema. New Zealand Journal of Agricultural Research, 34, 413-417.

Morris, C. A., Towers, N. R., \& Tempero, H. J. (1991b). Breeding for resistance to facial eczema in dairy cattle. Proceedings of the New Zealand Grassland Association, 53, 221-224. 
Nicol, A. M., \& Klotz, J. L. (2016). Ergovaline, an endophytic alkaloid. 2. Intake and impact on animal production, with reference to New Zealand. Animal Production Science, 56, 1775-1786.

Phua, S. H., Dodds, K. G., Morris, C. A., Henry, H. M., Beattie, A. E., Garmonsway, H. G., Towers, N. R., \& Crawford, A. M. (2009). A genome-screen experiment to detect quantitative trait loci affecting resistance to facial eczema disease in sheep. Animal Genetics, 40, 73-79.

Rogers, J. K., Walker, N. R., \& Young, C. A. (2016). The effect of endophytic fungi on nematode populations in summer-dormant and summer-active tall fescue. Journal of Nematology, 48, 87-94.

Siviero, I., Ferrante, S. M. R., Meio, I. B., Madi, K., \& Chagas, V. L. (2008). Hepatobiliary effects of cholic and lithocholic acids: experimental study in hamsters. Pediatric Surgery International, $24,325-331$.

Smith, B. L., \& Gravett, I. M. (1986). Uniformity of response of identical twin cattle to sporidesmin intoxication. New Zealand Veterinary Journal, 34,, 217-219.

Smith, B. L., \& O'hara, P. J. (1978). Bovine photosensitization in New Zealand. New Zealand Veterinary Journal, 26, 2-5.

Spivey, J. R., Bronk, S. F., \& Gores, G. J. (1993). Glycochenodeoxycholate-induced lethal hepatocellular injury in rat hepatocytesrole of ATP depletion and cytosolic-free calcium. Journal of Clinical Investigation, 92, 17-24.

Trottier, J., Bialek, A., Caron, P., Straka, R. J., Heathcote, J., Milkiewicz, P., \& Barbier, O. (2012). Metabolomic profiling of 17 bile acids in serum from patients with primary biliary cirrhosis and primary sclerosing cholangitis: A pilot study. Digestive and Liver Disease, 44, 303-310.

Wishart, D. S., Jewison, T., Guo, A. C., Wilson, M., Knox, C., Liu, Y., Djoumbou, Y., Mandal, R., Aziat, F., Dong, E., Bouatra, S., Sinelnikov, I. A., Xia, J., Liu, P., Yallou, F., Bjorndahl, T., Perez-Pineiro, R., Eisner, R., Allen, F., Greiner, R., \& Scalbert, A. (2013). HMDB 3.0-The Human Metabolome Database in 2013. Nucleic Acids Research, 41, 801-807.

Woolbright, B. L., Dorko, K., Antoine, D. J., Clarke, J. I., Gholami, P., Li, F., Kumer, S. C., Schmitt, T. M., Forster, J., Fan, F., Jenkins, R. E., Park, B. K., Hagenbuch, B., Olyaee, M., \& Jaeschke, H. (2015). Bile acid-induced necrosis in primary human hepatocytes and in patients with obstructive cholestasis. Toxicology and Applied Pharmacology, 283, 168-177.

Woolbright, B. L., Li, F., Xie, Y. C., Farhood, A., Fickert, P., Trauner, M., \& Jaeschke, H. (2014). Lithocholic acid feeding results in direct hepato-toxicity independent of neutrophil function in mice. Toxicology Letters, 228, 56-66.

Worley, B., \& Powers, R. (2013). Multivariate analysis in metabolomics. Current Metabolomics, 1,, 92-107.

Zhang, Y. C., Hong, J. Y., Rockwell, C. E., Copple, B. L., Jaeschke, H., \& Klaassen, C. D. (2012). Effect of bile duct ligation on bile acid composition in mouse serum and liver. Liver International, 32, 58-69. 